\title{
Cladistics
}

\section{Ending a decade of deception: a valiant failure, a not-so-valiant failure, and a success story}

\author{
Daniel R. Brooks ${ }^{\mathrm{a}, *}$, Ashley P. G. Dowling ${ }^{\mathrm{b}}$, Marco G. P. van Veller ${ }^{\mathrm{c}}$ and Eric P. Hoberg ${ }^{\mathrm{d}}$ \\ ${ }^{a}$ Department of Zoology, University of Toronto, M5S $3 G 5$ Toronto, Ontario, Canada \\ ${ }^{b}$ Museum of Zoology and Department of Ecology and Evolutionary Biology, University of Michigan, 1109 Geddes Avenue, \\ Ann Arbor, MI 48109, USA \\ ${ }^{c}$ Biosystematics Group, Wageningen University, Generaal Foulkesweg 37, 6703 BL Wageningen, the Netherlands \\ ${ }^{d}$ US National Parasite Collection and the Parasite Biology, Epidemiology and Systematics Laboratory, US Department of Agriculture, \\ Agricultural Research Service, BARC East 1180, 10300 Baltimore Avenue, Beltsville, MD 20705, USA
}

Accepted 12 December 2003

\begin{abstract}
Prior studies involving two methods, Brooks Parsimony Analysis (BPA) and TreeMap, have found BPA to be the more reliable method. Recent criticisms leveled at these studies argue that the tests were unfairly created and biased in favor of BPA. The authors of a recent critique offered new exemplars to demonstrate flaws in BPA, plus a simple fix to correct the flaws found in TreeMap. A re-evaluation of their exemplars clearly shows that the authors' calculations are incorrect, their understanding of the methods is lacking, and that their simple fix does not work. Additional analyses using TreeMap 2.02 are run to show that TreeMap 2.02, like TreeMap 1.0, cannot adequately deal with widespread parasites, contrary to the claims of its supporters. Furthermore, the exemplars corroborate previous findings that BPA, when calculated correctly, is more reliable than TreeMap1.0 and TreeMap 2.02 and therefore the method of choice in coevolutionary and biogeographic studies.
\end{abstract}

(c) The Willi Hennig Society 2004.

Siddall and Perkins (2003) criticized a simulation study by Dowling (2002) purporting to show that BPA (Brooks, 1981) was more reliable than TreeMap 1.0 in assessing the fit of parasite phylogenies to host phylogenies. Their critique comprised: (1) a presentation of exemplars, based on known (Cressey et al., 1983; Wiley, 1986, 1988a,b; Brooks, 1990) shortcomings of the original formulation of BPA, and empirical examples, to show flaws in BPA, coupled with (2) a proposed "fix" of TreeMap 1.0 to render the shortcomings found by Dowling (2002) moot; and (3) assertions that BPA is flawed philosophically, because it is a likelihoodist approach, and that the advocates of BPA do not understand parsimony. Herein, we show that: (1) all of their exemplars and examples,

*Corresponding author.

E-mail address: dbrooks@zoo.utoronto.ca when calculated correctly, support Dowling's (2002) assertion that BPA is more reliable than TreeMap 1.0 and 2.02; (2) their "fix" of TreeMap, although similar to the duplication rule proposed by Brooks (1990) to fix problems in the original formulation of BPA, serves to make TreeMap more similar to, but not more reliable than, BPA; and (3) their assertions about parsimony and likelihood approaches are confused at best. Siddall and Perkins' misrepresentation of the properties of both BPA and TreeMap is, moreover, not an isolated incident, but only the most recent episode in more than a decade of published reports in which: (1) a caricature of the original formulation of BPA is condemned; (2) the current version of BPA, proposed in 1990, is ignored; and (3) successive versions of Component Analysis, promoted as original contributions, converge more and more on the properties of BPA. 


\section{Siddall and Perkins miscalculated their exemplars and examples}

One of Siddall and Perkins' major complaints regarding Dowling's (2002) study is the creation of the test trials; they accused him of intentionally designing tests in favor of BPA over TreeMap. Siddall and Perkins therefore tried to provide exemplars intentionally designed to reveal supposed flaws in BPA, while highlighting the superior properties of TreeMap. Siddall and Perkins' exemplars, however, actually highlighted their apparent misunderstanding of both BPA and TreeMap, and, when calculated correctly, reinforce Dowling's (2002) conclusion that BPA is the more reliable method. In their exemplars $2 \mathrm{a}$ and $2 \mathrm{~b}$, for example, BPA actually finds the same most parsimonious explanation as TreeMap (Figs 1 and 2). Their exemplars $2 \mathrm{c}$ and 3 portray a parasite clade colonizing a

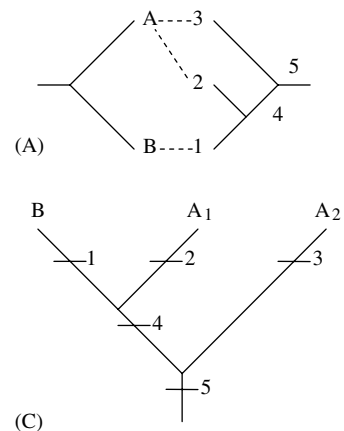

(B)

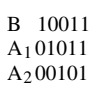

$\mathrm{A}_{2} 00101$

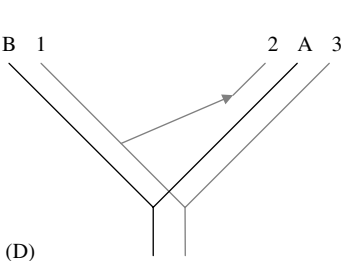

Fig. 1. Recalculation of BPA for Siddall and Perkins' exemplar 2a. (A) Host-parasite associations and phylogenies. (B) BPA matrix. (C) BPA phylogeny. (D) Most parsimonious BPA reconstruction. Arrows indicate host-switching events and colonizations and solid circles indicate points of co-speciation.
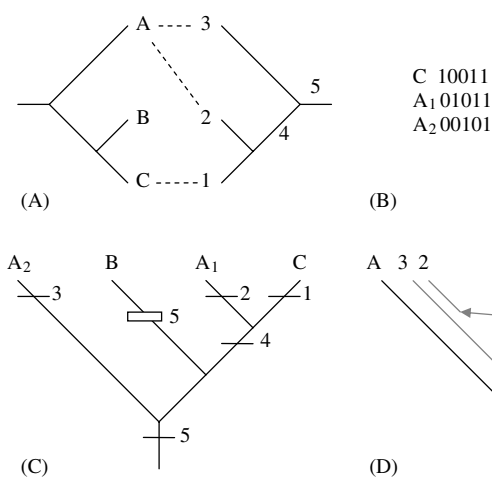

(B)

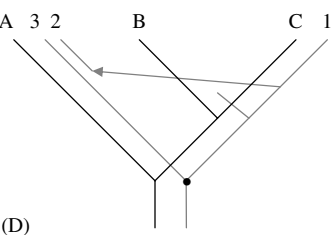

Fig. 2. Recalculation of BPA for Siddall and Perkins' exemplar 2b. (A) Host-parasite associations and phylogenies. (B) BPA matrix. (C) BPA phylogeny. Empty rectangle represents a character loss. (D) Most parsimonious BPA reconstruction. Arrows indicate host-switching events and colonizations and solid circles indicate points of co-speciation.

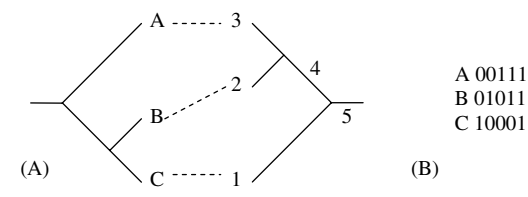

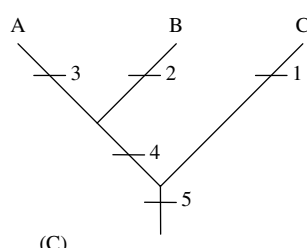

(C)

Fig. 3. Recalculation of BPA for Siddall and Perkins' exemplar 2c. (A) Host-parasite associations and phylogenies. (B) BPA matrix. (C) BPA phylogeny. (D) Most parsimonious BPA reconstruction. Arrows indicate host-switching events and colonizations and solid circles indicate points of co-speciation.

non-basal member of a host clade, then producing a descendant parasite which switches to one of the more basal, unoccupied, members of the host clade. When exemplar $2 \mathrm{c}$ is recalculated, BPA again obtains the same reconstruction as TreeMap (Fig. 3), although Siddall and Perkins claimed it never could do so. BPA also finds the same most parsimonious reconstruction as TreeMap for exemplar 3 (Fig. 4), which involves a late colonization with host switches back and forth across the host phylogeny.

Siddall and Perkins then presented empirical examples involving HIV and some of its relatives inhabiting primates (Mindell et al., 1995), and a small number of Plasmodium spp. and their vertebrate intermediate hosts (Perkins and Schall, 2002). The most parsimonious TreeMap reconstruction for the HIV/Primate example involves five co-speciations, four host switches, one duplication, and five sorting events for a total of what Siddall and Perkins call 10 ad hoc hypotheses. BPA does not consider all explanations for departures from cospeciation to be ad hoc, as we discuss below, but in this section we will follow the TreeMap conventions. Siddall and Perkins claimed that the most parsimonious BPA solution finds the same five co-speciations, but postulates 13 additional ad hoc hypotheses. In fact, BPA finds the same results as TreeMap (Fig. 5). Furthermore, BPA identifies the host switch between sister taxa, Pan and Homo, which Siddall and Perkins claimed is a solution "methodologically impossible for BPA to yield." In the Plasmodium/vertebrate example, Siddall and Perkins asserted that TreeMap yields five co-speciations and 12 ad hoc events (seven host switches, three duplications, and two lineage sorting events); whereas BPA finds six co-speciations but 23 ad hoc events (four host switches, four duplications, and 15 lineage sorting events). BPA actually produces seven co-speciations and multiple 

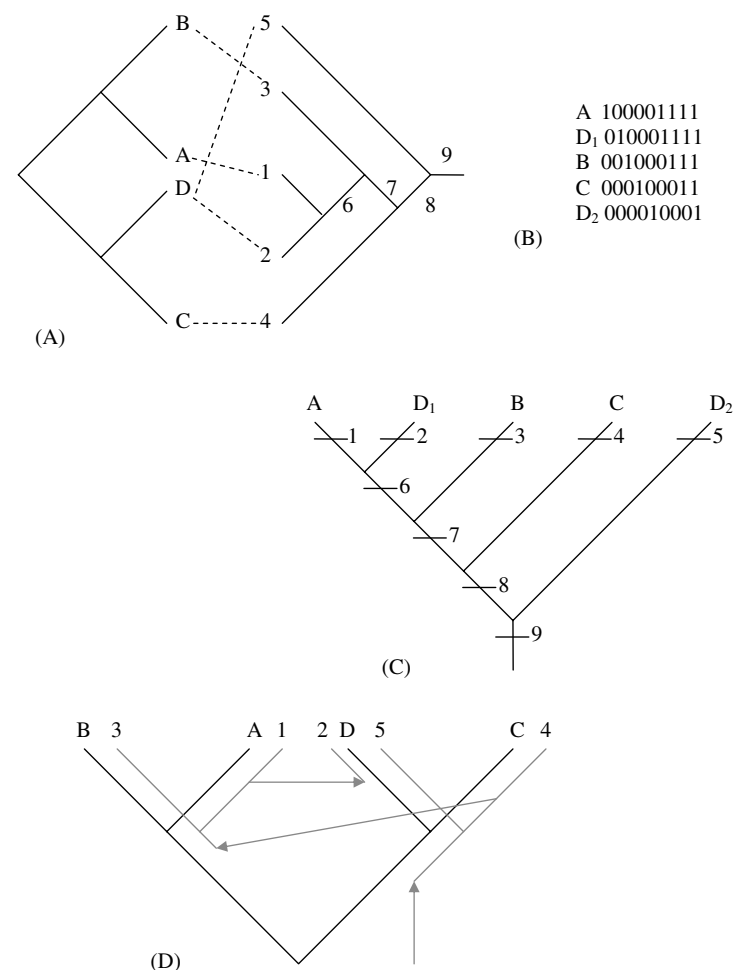

Fig. 4. Recalculation of BPA for Siddall and Perkins' exemplar 3. (A) Host-parasite associations and phylogenies. (B) BPA matrix. (C) BPA phylogeny. (D) Most parsimonious BPA reconstruction. Arrows indicate host-switching events and colonizations and solid circles indicate points of co-speciation.

equally parsimonious combinations of 13 ad hoc events, the default being two duplications, six host switches, and five sorting events (Fig. 6). An exhaustive search of every possible reconstruction in TreeMap 1.0 found nothing close to the Siddall and Perkins tree, and TreeMap 2.02 produced no reconstruction similar to their result. TreeMap 1.0 actually finds seven, not five, co-speciation events, just as does BPA, and the most parsimonious reconstruction proposes two independent sets of events. In one set, four reconstructions involve one duplication, seven host switches, and five extinctions, while in the other set six reconstructions involve two duplications, six host switches, and five sorting events. All reconstructions therefore require 13 ad hoc events, the same number as BPA. TreeMap 2.02, using Jungles, found four reconstructions, each also requiring seven co-speciations with 13 ad hoc events. It eludes us as to how Siddall and Perkins obtained their TreeMap result. Their preference for a reconstruction that posits fewer co-speciation events than either BPA or TreeMap points to an apparent lack of understanding of the goal of TreeMap. TreeMap is designed to minimize ad hoc explanations, but only after maximizing co-speciation events (Page, $1994 a, b)$. In an attempt to minimize total events, it is possible to create a pattern that depicts fewer events than either BPA or TreeMap reconstructions, but only if one is willing to eliminate some data from the input cladograms. We return to these points below.

BPA thus performs at least as well, and sometimes better, than TreeMap 1.0 and TreeMap 2.02 for Siddall and Perkins' exemplars and examples. Since those exemplars were created intentionally to highlight the shortcomings of BPA, they provide a strong corroboration of Dowling's (2002) contention that BPA is more reliable than TreeMap.

\section{Siddall and Perkins' "Fix" of TreeMap 1.0 does not work}

Siddall and Perkins contended that Dowling (2002) should not have included any test trials involving widespread parasites because TreeMap cannot handle them (Page, 1994b). We believe that a study intended to test the reliability of methods should, in fact, focus on situations in which the methods fail, not those in which they excel. To help TreeMap compete better with BPA, Siddall and Perkins proposed a "fix". This is accomplished by giving a parasite species a different designation each time it inhabits a different host, effectively turning one parasite into multiple sister taxa. TreeMap 1.0 specifically cannot handle widespread parasites due to host switching without speciation, and Siddall and Perkins's fix effectively turns a host-switch without speciation (one event) into a host switch with speciation (two events), which TreeMap can handle. Although they did not acknowledge it, this is precisely the way in which the current formulation of BPA handles widespread taxa (Brooks, 1990). Siddall and Perkins stated that TreeMap 2.02 does not suffer from this problem, but we disagree. The following two examples (Figs 7 and 8) are from Dowling's (2002, trials 4 and 14) study, featuring host switches without speciation. When these trials, or any other examples involving a host switch without speciation, are analyzed using TreeMap 1.0, they fail to find the correct pattern (Dowling, 2002). TreeMap 2.02 does not fare much better. The TreeMap 2.02 analysis of trial 4 (one host switch) produces seven reconstructions, three of which are considered most optimal, with a cost of four. In reality, two of the reconstructions have one host switch and one sorting event, while the third has a duplication and two sorting events. Apart from indicating that TreeMap 2.02 cannot count, and does not realize that $2<3$, these reconstructions contain one unnecessary sorting event.

As if these problems weren't enough, when the actual reconstructions are observed (Fig. 7B) it is apparent that for some reason host $\mathrm{H}$ does not have an associated parasite, even though it was entered into the analysis as having parasite VIII. Furthermore, the host switch, which should have been without speciation by parasite VIII from host $\mathrm{H}$, is now interpreted as a host switch with speciation. The same pattern is found in the 


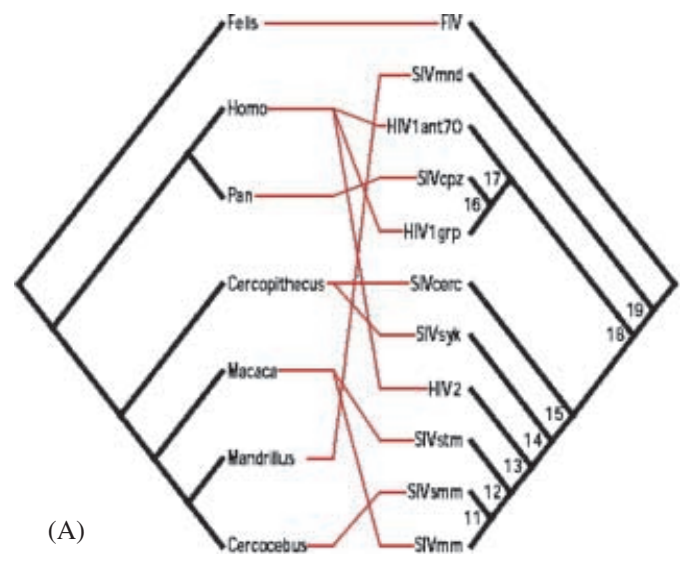

\begin{tabular}{|r|l|}
\hline $\mathrm{A}=$ & Cercocebus \\
\hline $\mathrm{B}=$ & Mandrillus \\
\hline $\mathrm{C}=$ & Macaca \\
\hline $\mathrm{D}=$ & Cercopithecus \\
\hline $\mathrm{E}=$ & Pan \\
\hline $\mathrm{F}=$ & Homo \\
\hline $\mathrm{G}=$ & Felis \\
\hline
\end{tabular}

(B)

\begin{tabular}{|l|l|l|l|l|l|}
\hline $\mathrm{C}_{1}$ & 10000 & 00000 & 01111 & 10011 & 1 \\
\hline $\mathrm{A}$ & 01000 & 00000 & 01111 & 10011 & 1 \\
\hline $\mathrm{C}_{2}$ & 00100 & 00000 & 00111 & 10011 & 1 \\
\hline $\mathrm{F}_{1}$ & 00010 & 00000 & 00011 & 10011 & 1 \\
\hline $\mathrm{D}_{1}$ & 00001 & 00000 & 00001 & 10011 & 1 \\
\hline $\mathrm{D}_{2}$ & 00000 & 10000 & 00000 & 10011 & 1 \\
\hline $\mathrm{F}_{2}$ & 00000 & 01000 & 00000 & 01111 & 1 \\
\hline $\mathrm{E}$ & 00000 & 00100 & 00000 & 01111 & 1 \\
\hline $\mathrm{F}_{3}$ & 00000 & 00010 & 00000 & 00111 & 1 \\
\hline $\mathrm{B}$ & 00000 & 00001 & 00000 & 00001 & 1 \\
\hline & 00000 & 00000 & 10000 & 00000 & 1 \\
\hline
\end{tabular}
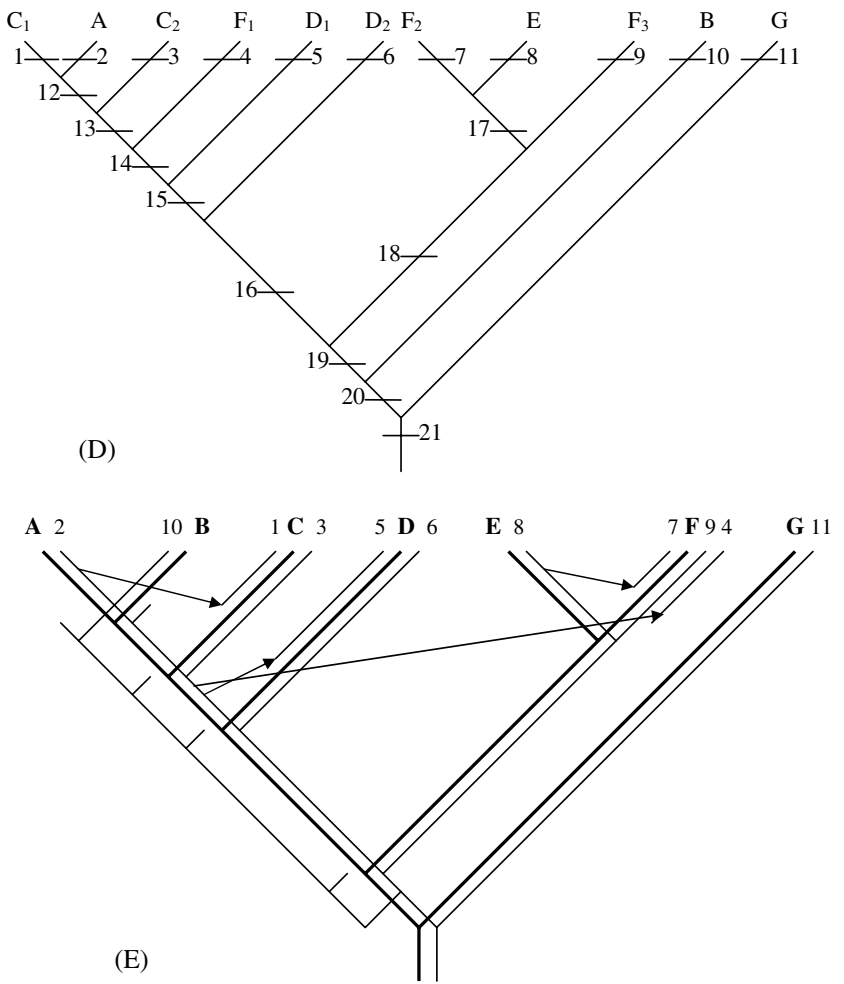

Fig. 5. Recalculation of BPA for Siddall and Perkins' HIV exemplar. (A) Host-parasite associations and phylogenies. (B) Host key. (C) BPA matrix. (D) BPA phylogeny. (E) One of the most parsimonious BPA reconstructions. Arrows indicate host-switching events and colonizations and solid circles indicate points of co-speciation.

analysis of trial 14 (Fig. 8), which involved two separate host switches without speciation; but the results for this trial are even more perplexing. Ten reconstructions are obtained and two with a cost of four are deemed most optimal (Fig. 8B). In these results, only one host, D, has two parasites, while host $\mathrm{G}$, which should have had two parasites, now has only one. According to the input data, hosts $\mathrm{D}$ and $\mathrm{E}$ have parasite $\mathrm{V}$, but as a result of the analysis, host $\mathrm{E}$ has lost it. Finally only one host switch is identified, when there should be two, and that one is displayed as a host switch with speciation as opposed to the switch without speciation that actually occurred. This being the case, we are at a loss as to how Siddall and Perkins can consider TreeMap 2.02 to have solved the widespread parasite problem. It is obvious that TreeMap 2.02 still does not handle instances of host switching without speciation, and the fix must be necessary for TreeMap 2.02 .

\section{Finally, Siddall and Perkins did not implement BPA's duplication convention correctly}

If the widespread parasite is due to host switching without speciation, the fix helps TreeMap identify the correct general pattern, but not all widespread parasite distributions are the result of host switching without speciation. The exemplar shown below highlights a situation involving widespread parasites in which the fix fails to help TreeMap identify the correct pattern uncovered by BPA. The host-parasite association involves four hosts and three parasites (Fig. 9), one of which (parasite II) is found on two different hosts (A and C). In order to apply the TreeMap fix, parasite II is split into IIa and IIb. TreeMap then obtains two equally optimal reconstructions, each involving a host switch and sorting event (Fig. 10). What if, as proposed by Hennig (1966), Brooks (1979, 1981, 1990), Wiley (1986, 1988a,b), Johnson and Clayton (2003), and Johnson et al. (2003), widespread species II is due to host speciation without parasite speciation (Fig. 11)? The fix created by Siddall and Perkins then leads to erroneous reconstructions, whereas BPA (Fig. 12) continues to accurately reflect the pattern portrayed in Fig. 11. If the fix is applied to TreeMap 2.02, the same results as found with TreeMap 1.0 (Fig. 9) are obtained.

BPA performs more reliably than TreeMap over a wider range of possibilities because it does not require all widespread parasites to be the result of host 


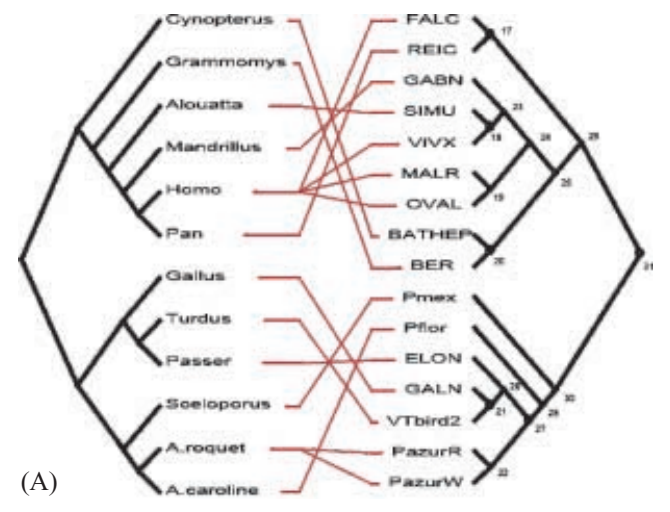

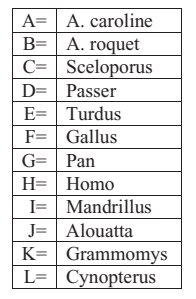

(B)
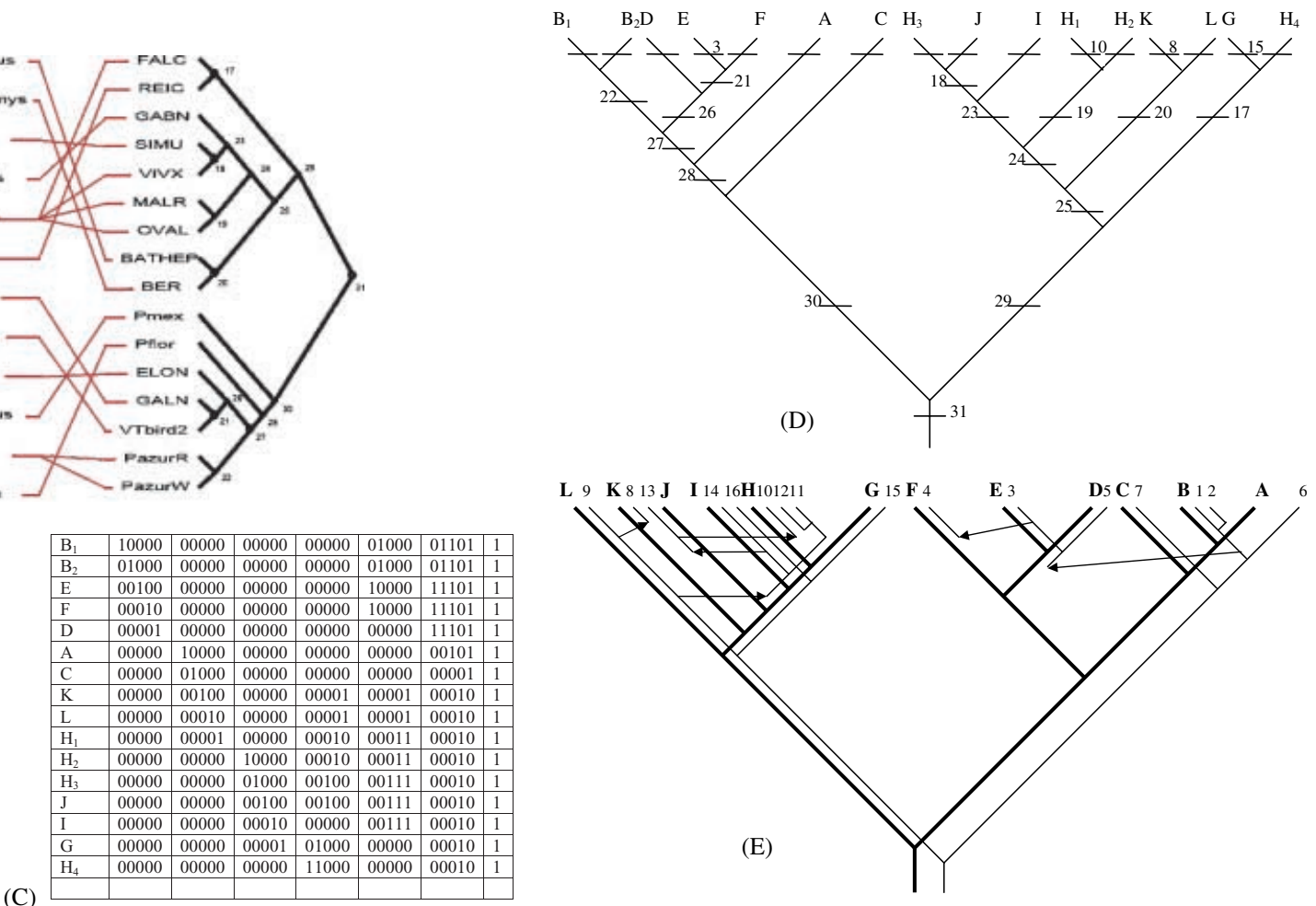

Fig. 6. Recalculation of BPA for Siddall and Perkins' Plasmodium exemplar. (A) Host-parasite associations and phylogenies. (B) Host key. (C) BPA matrix. (D) BPA phylogeny. (E) One of the most parsimonious BPA reconstructions. Arrows indicate host-switching events and colonizations and solid circles indicate points of co-speciation.

switching events. Ironically, the general situation in which the TreeMap fix fails is one in which a widespread parasite species provides additional cospeciation support (Hennig, 1966; Brooks, 1979, 1981, 1990). This is precisely the reason that BPA of two groups of nematodes inhabiting Great Apes showed that TreeMap analysis of one of the clades had produced a less than parsimonious result and had actually underestimated the number of co-speciation events supported by the data (Brooks and McLennan, 2003). Siddall and Perkins neglected to mention those findings, despite the fact that their own two empirical examples involved some parasites inhabiting primates, and that the study by Brooks and McLennan (2003) was published in Cladistics.

\section{Dowling's simulations were not biased towards BPA}

From the beginning of post-Hennigian discussions about co-evolution and phylogeny, Brooks $(1979,1981)$ consistently advocated the development of methods that did not rely on an a priori assumption that the true host phylogeny was known, or that there had been any degree of co-speciation, arguing that such a priori assumptions would weaken inferences (results are more robust if you find what you are looking for using a method that does not assume that it even exists) (for extensive reviews, see Brooks and McLennan, 1991, 1993, 2002). Hennig (1966, pp. 174-180) originally pointed out the potential circularity in assuming the host phylogeny, then assuming maximum co-speciation, then fitting parasites to the host phylogeny, finally claiming to have found evidence of co-speciation (Hennig did not use the term co-speciation, which was proposed by Brooks, 1979). Hennig suggested basing comparisons on independently derived parasite phylogenies. Brooks (1979) showed that, because any given parasite species can exhibit a wide range of character associations with their hosts, even mapping an independently derived parasite phylogeny on a host phylogeny would not be a robust test of anything.

The weakest possible test of phylogenetic associations between hosts and parasites therefore utilizes only a single parasite clade. In such cases, BPA reproduces the taxon-host cladogram, an accurate map of the sequence of speciation events supported by the parasite tree. Providing any sort of explanation for the hosts requires that the researcher choose a host phylogeny assumed to be true, and then optimize the parasite phylogeny onto it 

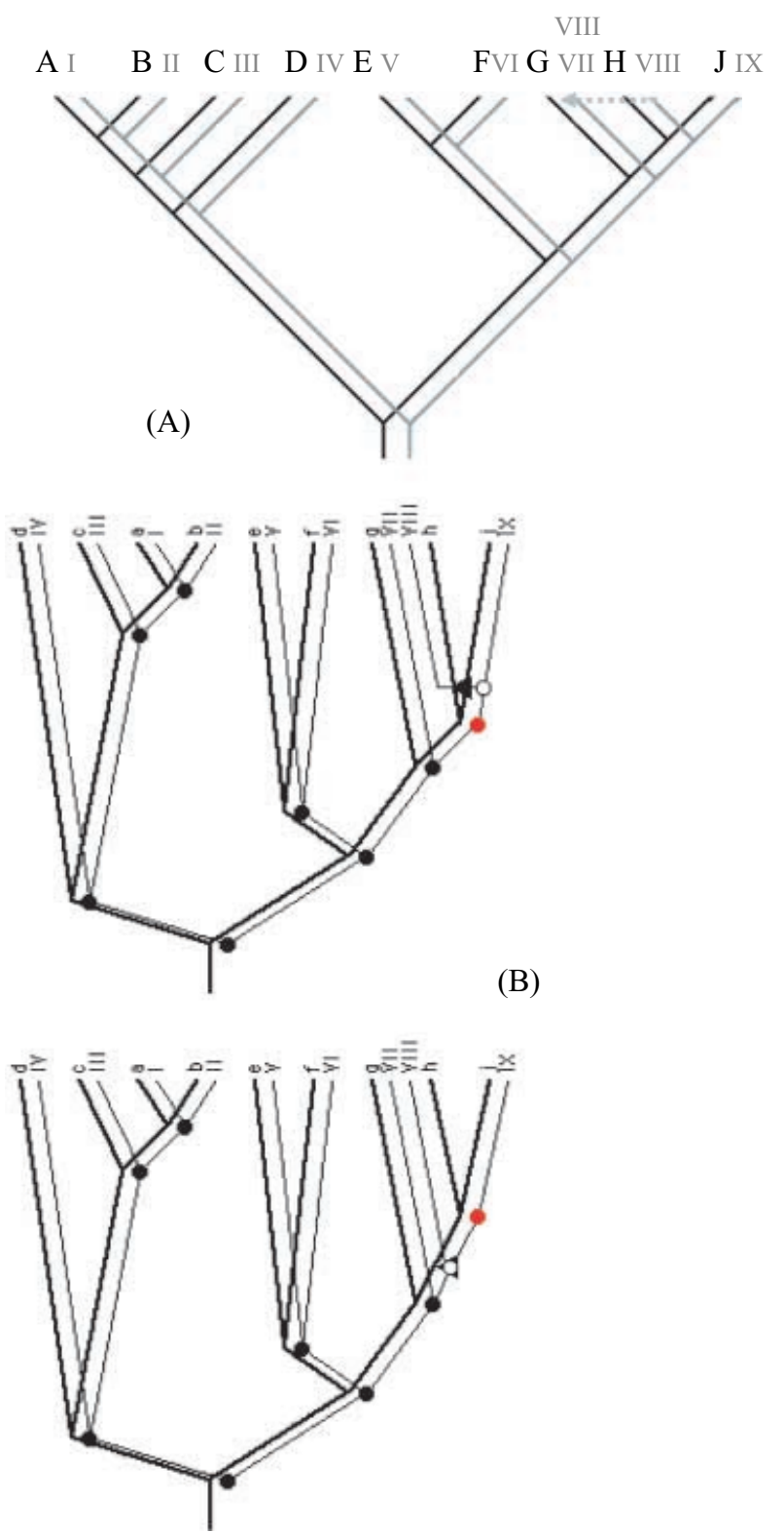

Fig. 7. Re-analysis of Trial 4 from Dowling (2002) using TreeMap 2.02. (A) Host-parasite associations and phylogenies. (B) Two most optimal reconstructions. The inability of TreeMap 2.02 to handle widespread parasites is evidenced by the deletion of parasite VII from host $\mathrm{h}$, the extraneous sorting event, and the host switch with speciation that should have been without speciation.

(Fig. 13). The parasite phylogeny (Fig. 13B) contains four speciation events. BPA is required to provide four explanations; additional explanations are ad hoc. If we know the host phylogeny (Fig. 13A), we can infer co-speciation for three of the parasite speciation events (circles in Fig. 13B) and speciation by host switching for the other (triangle in Fig. 13B), without assuming co-speciation a priori. If host species B is the result of a peripheral isolates speciation (i.e. speciation by
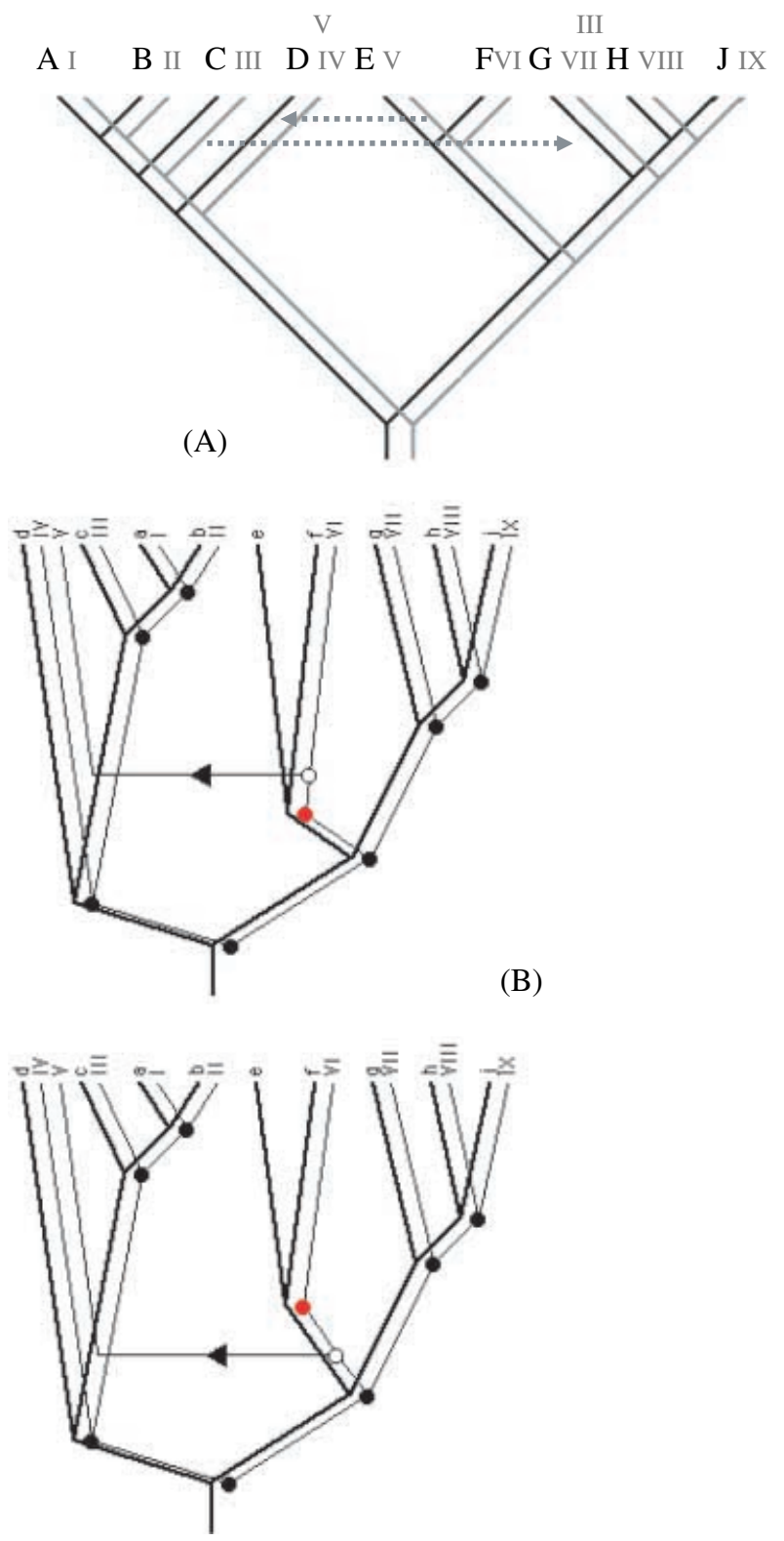

Fig. 8. Re-analysis of Trial 14 from Dowling (2002) using TreeMap 2.02. (A) Host-parasite associations and phylogenies. (B) Two most optimal reconstructions. The inability of TreeMap 2.02 to handle widespread parasites is evidenced by the deletion of parasite $\mathrm{V}$ from host $\mathrm{e}$ and parasite III from host $\mathrm{g}$, the extraneous sorting event, one of the two host switches is missing, and the present host switch with speciation should have been without speciation.

dispersal) event in which members of the parasite clade did not participate, the absence of any member of the parasite group in host B needs no explanation involving the parasite clade. Cetaceans, for example, have very few parasites that are closely related to parasites inhabiting hippopotami, but this is most likely due to the failure of parasite lineages to move into marine habitats than to co-speciation and extinction in all those 


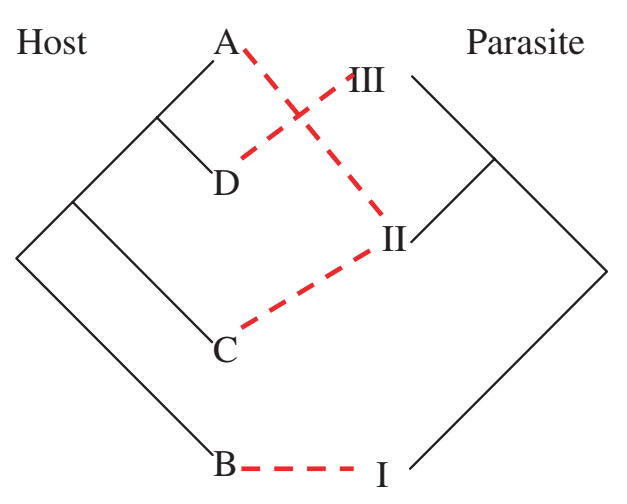

Fig. 9. Host (A-D) and parasite (I-III) associations and phylogenies.
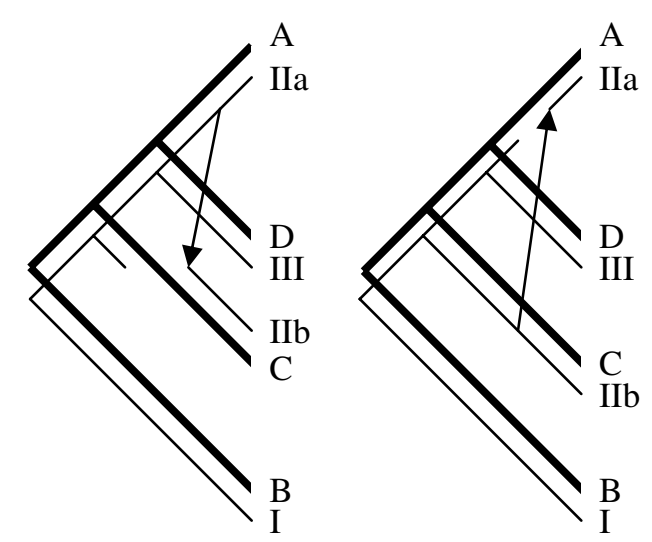

Fig. 10. Two most optimal TreeMap 1.0 reconstructions based on host-parasite relationships (Fig. 9) after the Siddall and Perkins "fix" is applied. Both explanations involve a single host switch and a sorting event.

lineages. Four events, four explanations, nothing ad hoc (TreeMap would call the episode of speciation by host switching an ad hoc event). If, however, we assume maximum co-speciation a priori, we obtain a result that requires an ad hoc explanation, extinction, for the absence of a parasite species inhabiting host B that is congruent with the host phylogeny (Fig. 9C). This is precisely what tree reconciliation does, contrary to Siddall and Perkins' assertion that TreeMap has nothing to do with using parasites to assess host phylogeny. Dowling's results are thus all the stronger for having forced BPA to perform in the arena of TreeMap, in which it may be forced into less than most parsimonious explanations.

BPA was designed to provide an explicit and accurate description of parasite speciation events in the context of host associations. This is accomplished by asking if three or more parasite clades show any congruence (co-speciation), and only then asking what that might mean in terms of host relationships. Brooks and McLennan (1991) discussed this at length, and Brooks
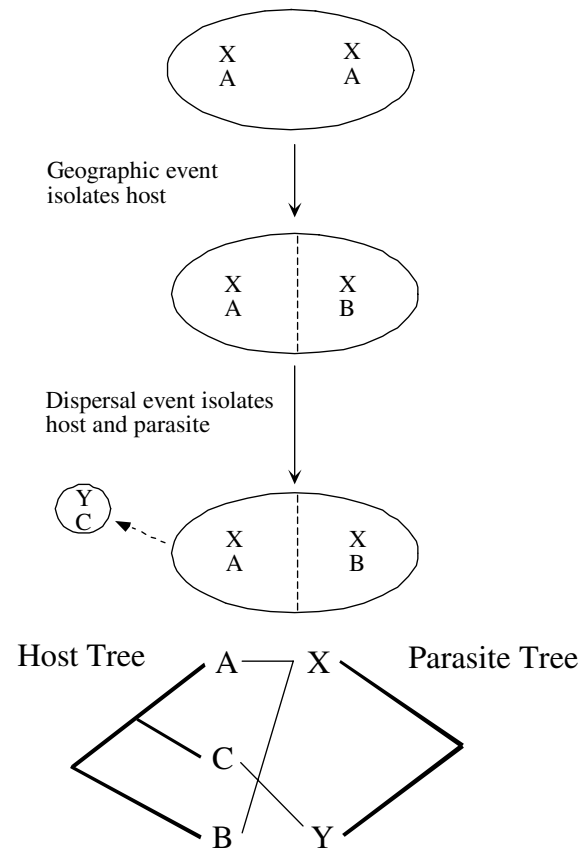

Fig. 11. Hypothetical scenario for speciation in hosts and parasites (taken from Johnson and Clayton, 2003). A geographic event divides host species A resulting in speciation forming hosts A and B. Parasite $\mathrm{X}$ does not speciate and is now found widespread on host $\mathrm{A}$ and $\mathrm{B}$. Members of host species A then colonize an isolated area that results in speciation of both host and parasite, forming host $\mathrm{C}$ and parasite $\mathrm{Y}$.

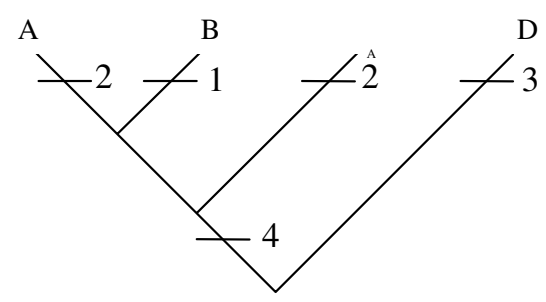

Fig. 12. Most parsimonious BPA phylogeny indicating the host duplication without subsequent parasite speciation.

et al. (2001), Brooks and McLennan (2002, 2003), and Dowling et al. (2003) recently generalized this as the "Threes Rule" rule. Figure 14 shows how this can lead us to a most parsimonious inference of extinction for the parasite clade in Fig. 13. Consider three parasite clades, one of which (Fig. 14B) is the same as Fig. 13B. The parasite-host cladogram for the other two parasite clades (Figs 14C, D) is identical to the host phylogeny (Fig. 14A). The host cladogram generated by BPA (Fig. 14E) supports the inference that absence of a member of parasite clade one from host B, whose relationships are congruent with the host phylogeny, is the result of extinction. This is because an explanation of speciation by host switching by members of two different parasite clades is now less parsimonious (two 


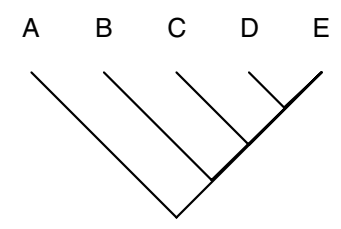

(A)

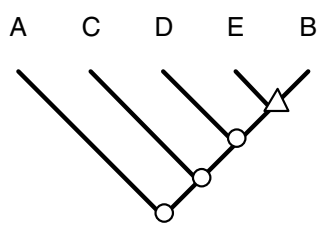

(B)

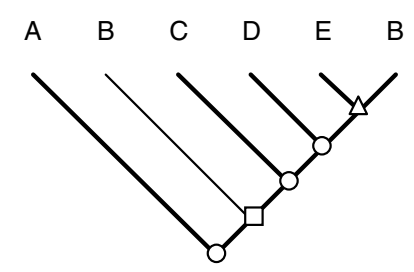

(C)

Fig. 13. (A) Host phylogeny. (B) Parasite phylogeny converted into parasite-host cladogram by replacing parasite names with host letters. Circles $=$ co-speciation episodes (speciation events in the parasites that are congruent with the host phylogeny); triangle = episode of peripheral isolates speciation (speciation by host switching). Note that each observed event on the parasite phylogeny has an explanation. (C) Parasite-host cladogram from (B) optimized onto host phylogeny from (A) under the assumption of maximum co-speciation. Circles = co-speciation episodes (speciation events in the parasites that are congruent with the host phylogeny); triangle = episode of peripheral isolates speciation (speciation by host switching); square $=$ ad hoc inference of extinction. Note that we now postulate five explanations for four observed events, making this solution less parsimonious than the one shown in (B).

ad hoc events) than an explanation of extinction (one ad hoc event). Note also that none of the explanations for the four observed nodes in the parasite phylogeny in Figs 13B, 14B is altered by this additional information. This supports the BPA interpretation that speciation by host switching on the part of the member of the parasite clade in Figs 13B, 14B is the most parsimonious inference of an observed feature of the data, not an ad hoc explanation.

The null expectation, stated explicitly by Brooks (1981), is that congruence among parasite clades will correspond to host phylogeny, but (1) knowledge of the host phylogeny is not needed to distinguish general from special elements if multiple parasite clades are analyzed; (2) general patterns of host relationships indicate co-speciation among the parasites, but they need not be congruent with host phylogeny (e.g. Hoberg, 1986; Hoberg and Adams, 1992; Hoberg, 1995; Hoberg et al., 1997; Hoberg and Adams, 2000; Hoberg and Klassen, 2002); and (3) the relationship between parasite speciation patterns in a BPA and host phylogeny can be tested by comparison with an independently generated host phylogeny and with biogeographic information (Hennig, 1966; Brooks, 1979, 1981, 1985, 1990; Brooks

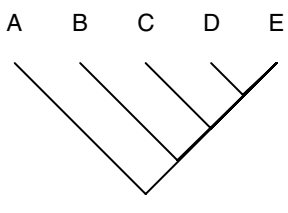

(A)

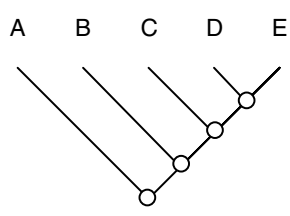

(C)

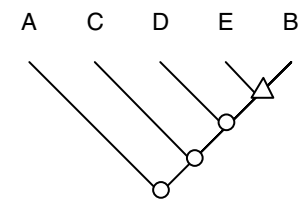

(B)

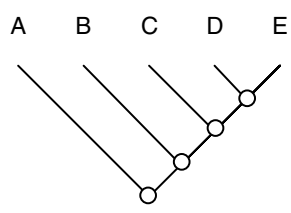

(D)

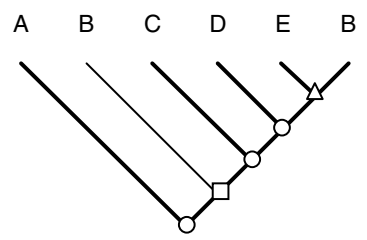

(E)

Fig. 14. (A) Host phylogeny. (B-D) Parasite phylogenies converted into parasite-host cladogram by replacing parasite names with host letters. Circles $=$ co-speciation episodes (speciation events in the parasites that are congruent with the host phylogeny); triangle $=$ epiepisode of peripheral isolates speciation (speciation by host switching). Note that each observed event on the parasite phylogeny has an explanation. (E) Parasite-host cladogram from BPA of (B-D). Circles $=$ co-speciation episodes (speciation events in the parasites that are congruent with the host phylogeny); triangle $=$ episode of peripheral isolates speciation (speciation by host switching); squar$\mathrm{e}=$ ad hoc inference of extinction. In this case, inferring extinction of a parasite species from host B in (B) is more parsimonious than inferring two episodes of speciation by host switching in (C) and (D).

and McLennan, 1991, 1993, 2002). Siddall and Perkins' assertion that BPA is a method for using parasite phylogenies to derive host phylogenies is thus factually incorrect. BPA makes no a priori assumptions about the degree, or even existence, of co-speciation, actually being an assessment of co-speciation among parasites, with host relationships a byproduct of the search for general patterns of congruence among parasites. This is completely analogous to phylogenetic systematics, which is analysis of characters, not of taxa, searching for character congruence, which we then use in phylogenetic inference.

Siddall and Perkins' exemplars and examples demonstrate that even the weakest application of BPA outperforms TreeMap 1.0 and 2.02, and highlights some previously unreported shortcomings of TreeMap as well. Dowling's (2002) study, far from being an effort to favor BPA over TreeMap, was a scrupulous effort to make certain that TreeMap was not put at an undue disadvantage vis-à-vis BPA. 


\section{The philosophical basis of the debate}

Dowling's (2002) study utilized 62 trials. Siddall and Perkins highlighted the two trials in which neither primary BPA nor TreeMap obtained the correct answer because the "known" history was not the most parsimonious interpretation of the final pattern. Dowling did not criticize either method for this shortcoming, but Siddall and Perkins used those cases to criticize Dowling's understanding of parsimony, and to characterize BPA as a likelihoodist exercise. Given that Siddall and Perkins did not produce the most parsimonious interpretation for any of their own exemplars and examples, the issue of what really is parsimony deserves some discussion.

McAllister (1996) suggested that scientists make decisions about the theories they support based partly on empirical and partly on aesthetic considerations. He furthermore noted that a sense of simplicity, or parsimony, was a common element of both. Discussions about phylogenetic methods of analysis of co-evolutionary systems parallel those involving methods of historical biogeographic analysis. In previous discussions, we have alluded to two different senses of parsimony in these discussions. The first category deals with ontological considerations; what is the nature of the system or, what is your a priori model of the nature of the system?

Advocates of TreeMap and its precursors claim the philosophical high ground in this regard, asserting that their model is more parsimonious than BPA. Superficially, this is so-asserting that the nature of coevolution is predominantly co-speciation, so much so that departures from strict co-speciation are rare enough to be ad hoc events, seems highly parsimonious. This is what led Van Veller and Brooks (2001) and Brooks and McLennan (2002) to portray the maximum co-speciation model as stemming from an ontology of simplicity (= a priori parsimony). BPA, by contrast, suggests that the only ad hoc explanations are those requiring more statements than the number of speciation events indicated by the parasite clades used as primary data. That is, phenomena other than strict co-speciation are not necessarily in need of ad hoc explanations. In fact, the only ad hoc assertions in BPA as it has existed since 1990 are inferences of extinction. This apparently non-parsimonious view of the nature of co-evolution, portrayed by Van Veller and Brooks (2001) and Brooks and McLennan (2002) as stemming from an ontology of complexity (= a posteriori parsimony), should not offend the empirical sensibilities of advocates of TreeMap, since they have repeatedly modified their computer programs to incorporate the broader range of possibilities permitted by BPA. There must therefore be something about BPA that offends their aesthetic sensibilities.
One possible reason for this confusion is that the maximum co-speciation model may not be as parsimonious as it seems. If co-evolution is predominantly co-speciation, parasite species must be predominantly narrowly specialized on host resources that show a $1: 1$ mapping with the phylogenetic diversification of the hosts. Colonization of new hosts is accomplished only on the rare occasions that a given parasite evolves the ability to colonize a new host, in effect becoming a generalist. The maximum co-speciation model assumes this is such a rare occurrence that hosts of widespread species that are incongruent with the host phylogeny can be deleted from the analysis (by Assumption 2 in TreeMap 1.0 or by Jungles in TreeMap 2.02). Even more rare is a situation in which the parasite expands its host range and then becomes extinct in the host in which it co-speciated, thereby appearing to have arisen through a host switch (first discussed by Brooks, 1981). This assumption allows Jungles to assign a higher cost to "host switching" (which BPA considers to be a single event) than to "lineage duplication + sorting" (which BPA considers to be at least two events). Next, any situation in which one could enhance the fit to a co-speciation model by postulating an extinction event is taken de facto as evidence of extinction. Finally, lineage duplications are episodes of sympatric speciation (speciation within the same host species: Brooks and McLennan, 1993) which leads to two strictly co-speciating lineages, each of which is specialized on a host resource that shows a $1: 1$ mapping with the phylogenetic diversification of the hosts.

BPA does not presuppose that co-speciation occurs. When it does, however, it need be no more than the result of hosts and parasites experiencing the same allopatric speciation events solely as a by-product of their spatial proximity (Brooks, 1981). Furthermore, post-speciation host range expansion and speciation by host switching (a form of peripheral isolates speciation: Brooks and McLennan, 1993) require no more than contact (common or rare, respectively) with a host species having the same specialized resource as the host from which the colonization event took place. Because the resource can be synapomorphic, symplesiomorphic, or homoplasious with respect to all the host species inhabited, there are no a priori expectations with respect to the degree of phylogenetic relatedness of the hosts (Brooks and McLennan, 2002), so no a priori cost can be assigned to widespread species. The supposition that speciation by host switching is a regular, rather than ad hoc, feature of co-evolution, is also the reason that extinctions cannot be inferred from the absence, from a given host, of parasites of a single parasite clade, unless we assume maximum co-speciation (Fig. 13); robust inferences of extinctions can be made using BPA only if a minimum of three parasite clades are analyzed together (Brooks and McLennan, 1991, 1993, 2002; also 
Brooks et al., 2001)(Fig. 14). Finally, lineage duplications in BPA indicate episodes of sympatric speciation, just as with the maximum co-speciation model, but differ by not implying that any given episode of sympatric speciation need be followed by strict co-speciation in either lineage. Overall, then, the maximum co-speciation model is a priori more parsimonious than BPA in terms of the simplicity of allowed patterns. It is not quite so clear that it is a priori more parsimonious in terms of required mechanisms.

Also relevant to ontological considerations is the demonstration that a model is consistent with a wellcorroborated current theory (McAllister, 1996). The maximum co-speciation model is most consistent with orthogenesis (Brooks and McLennan, 1993, 2002; Brooks, 2003), not considered a well-corroborated current theory. Darwinism, considered a well-corroborated current theory, does not require maximum co-speciation; in fact, as a theory of historical contingency, it is more consistent with BPA. Most advocates of the maximum co-speciation school have been silent about the nature of the conceptual framework to which they subscribe, but some have been more forthcoming. Ebach and Humphries (2002, p. 427) criticized BPA for "taking an evolutionary stance..." (see also Ebach, 2001; Ebach et al., 2003), and Siddall and Perkins claimed that TreeMap 1.0 and 2.02 are used for "tree comparison" and not for "inference of host cladograms from parasite phylogenies and distributions." Such statements may explain why advocates of the maximum co-speciation and maximum vicariance research programs prefer the terms "lineage duplication", "lineage sorting", or "missing the boat" to the more standard terms "sympatric speciation", "extinction", and "non-response to a host speciation event", but the underlying ontological dilemma remains, despite denials that there is any ontological basis to the disputes (Ebach and Humphries, 2002; Ebach et al., 2003). If "tree comparisons" are not for the purposes of (co)evolutionary inferences, what are they for?

Parsimony considerations also play a role at the epistemological level, affecting the way in which basic data are analyzed and the manner in which explanations are inferred from an analysis. Siddall and Perkins' own exemplars and examples demonstrate that, when the methods disagree, BPA provides more parsimonious results than TreeMap. This is due to two strengths of BPA: (1) strict adherence to Assumption 0 (Wiley, 1986, 1988a,b; Zandee and Roos, 1987), which states that all information from all input parasite-host cladograms must be used without modification or deletions, and the final host cladogram must be logically consistent with all input parasite-host cladograms (see also Brooks and McLennan, 2002), and (2) absence of restrictive a priori explanations for widespread parasites.

That TreeMap with the Siddall and Perkins' fix better mimics BPA cannot argue for BPA being a failure unless
TreeMap is a failure. We agree, however, that the original formulation of BPA was a valiant failure, in spite of being better than TreeMap, but not for the reasons proposed by Siddall and Perkins. Brooks (1981) assumed that the analytical problems associated with particular histories of host associations in particular clades would be overcome by analysis of multiple parasite clades, and many of them can be. Cressey et al. (1983), however, showed that combining the codes for all parasites associated with the same host (inclusive ORing) proposed by Brooks (1981), could create systemic problems for groups whose evolution had not been driven primarily by co-speciation. Three classes of potential problems associated with inclusive ORing have been discussed at length in at least three books (Brooks and McLennan, 1991, 1993, 2002) and in other venues (Hoberg et al., 1997; Brooks et al., 2001; Hoberg and Klassen, 2002). Siddall and Perkins did not address the fact that Brooks' (1990) modification of BPA, so-called secondary BPA, eliminated all problems associated with inclusive ORing, by eliminating inclusive ORing from the method. BPA without inclusive ORing never violates Assumption 0, so TreeMap can never be more parsimonious than BPA, and here we find a possible answer to the question of aesthetics.

A priori methods are designed to provide the most parsimonious solution possible, given the constraints of the model upon which the method is based. By invoking Assumption 0, BPA prohibits any modification of the input data and any analytical result that is logically inconsistent with any of the input data, opting for the most parsimonious result that does not violate Assumption 0. Alternatively, TreeMap maximizes co-speciation and only then minimizes the number of ad hoc events resulting from maximizing co-speciation; it is not designed to ask if there is an even more parsimonious solution, supporting less than maximal co-speciation. Advocates of TreeMap introduce a sense of a priori parsimony, stating that, if one assumes a priori that coevolution is generally a matter of co-speciation, then it is most parsimonious to maximize putative co-speciation events a priori. It is then more parsimonious to attach a higher "cost" (lower probability) to "sympatric speciation + extinction" (lineage duplication + sorting) than to "host switching". So long as the constrained solution is the same as the most parsimonious solution, the model is corroborated, but, more importantly, when the constrained solution is less than maximally parsimonious, we have evidence of specific or general refutations of the model. Because each modification of Component Analysis and its descendants, Reconciled Tree Analysis (RTA) and TreeMap 1.0 and 2.02, has converged on BPA, it is difficult not to conclude that analyses of empirical data by the very method designed to promote the maximum co-speciation model have consistently refuted it. 
Van Veller and Brooks (2001) proposed that the a priorist and a posteriorist methods each properly implemented an ontologically distinct research program, and thus should not be considered direct competitors epistemologically. Ebach and Humphries (2002), Page (2003b), and Siddall and Perkins (2003), however, rejected that proposal, and chose to attack BPA as a direct competitor with TreeMap and other $a$ priorist approaches. Van Veller and Brooks (2001) further suggested that, if one were not going to accept that TreeMap and BPA represented different methods each properly implementing an ontologically distinct research program, the performance of all methods should be judged against the post-1990 formulation of BPA. Studies by Van Veller et al. (1999, 2000, 2001, 2002) and Dowling (2002), and responses to critics of those studies (Van Veller and Brooks, 2001; Brooks, 2003; Dowling et al., 2003; Van Veller et al., 2003; this contribution) have shown unambiguously that if there is to be a single preferred method of pattern analysis in phylogenetic studies of biogeography and co-evolution, that method should be BPA as currently formulated.

McAllister (1996) suggested five other criteria for assessing the empirical adequacy of competing models: (1) internal consistency; (2) consistency with extant current empirical data; (3) empirical content; (4) novel prediction; and (5) explanatory power.

Van Veller and colleagues (Van Veller et al., 1999, 2000, 2001, 2002; Van Veller and Brooks, 2001) have provided the most extensive study of the degree of internal consistency among these methods. They discovered that all methods other than the post-1990 formulation of BPA exhibit internal inconsistencies under certain conditions. Ebach and Humphries (2002) objected to Van Veller and colleagues' findings, presenting an exemplar designed to show a case in which Assumption 0 analysis (by which they meant the original formulation of BPA, not the real Assumption 0 ) failed to be consistent, whereas Assumption 2 analysis was consistent using Van Veller and colleagues' own criteria. Van Veller et al. (2003) showed that when their exemplar was calculated correctly, Assumption 0 analysis was consistent and Assumption 2 analysis was not, providing additional support for Van Veller and colleagues' findings. We find that episode remarkably similar to Siddall and Perkins' attack on Dowling's work.

With regard to empirical content, Van Veller et al. (2000) recognized a priori and a posteriori methodologies in historical biogeography (the same methods are used in co-evolutionary studies) based upon whether they permit or prohibit modification of the input taxonarea (taxon-host) cladograms, respectively. Some a priori methods permit the modification of data in the parasitehost cladogram by deleting some occurrences of widespread parasites in hosts that are inhabited by (other) endemic parasites (of the same monophyletic group) or by the addition of hypothetical taxa ("missing species", "leaves", or "losses") that are assumed to be extinct. Van Veller et al. (1999, 2000, 2003; Green et al., 2002) discussed this in a historical biogeographic context:

\begin{abstract}
"For area D, the conflict is between widespread and endemic taxa. Assumption two resolves such conflict on behalf of endemics, eliminating area $\mathrm{D}$ (in parentheses) from association with areas BCG (Fig. 5j)." (Nelson and Ladiges, 1991, p. 479).

"If a node leads directly to one or more terminal taxon that is geographically widespread, and part of that distribution overlaps with that of another taxon, or taxa, then the widespread distribution is reduced to the non-overlapping geographic element." (Nelson and Ladiges, 1996; p. 3-4).

"... the problem may be analyzed under the assumption that all errors are due to missing species ..." (Nelson and Platnick, 1981, p. 417) and "If sampling error arises because of missing information, the missing information can be added to components 5 ! and 6 ! in order to convert them into components of the original concept." (Nelson and Platnick, 1981, p. 413).

“Taking Rosen's (1978) taxon cladograms and distributions and searching for the area cladogram that minimizes the number of leaves that must be added to reconcile the two fish cladograms with an area cladogram, we obtain the area cladogram in Figure 10a. This cladogram requires a total of 32 leaves added, 10 for Heterandria and 22 for Xiphophorus ..." and "These 32 leaves added correspond to at least 18 independent extinctions, ..." (Page, 1994a, p. 72).
\end{abstract}

TreeMap 1.0 and TreeMap 2.02 allow and encourage modification of the data based upon the researcher's expectations with respect to the associated (evolutionary) relationships between the parasites and the hosts. With TreeMap 1.0 such modification is applied when removing data (hosts) that are assumed to be the result of a host switch:

"The map associated with this reconstruction differs from the map for the reconciled tree, and is obtained by modifying the host set of each node that is ancestral to the parasite I by removing the host (a) that was acquired by host switching (Table 2)." (Page, 1994b, p. 159).

TreeMap 2.02 uses the same a priori modification of data through a weighting scheme with different "costs" that may overrule the cladogenetic information included in the data, thus permitting Assumption 0 to be violated if the data do not conform to the model. In some cases, modifications of TreeMap 1.0 and 2.02 result in not using all the information in the data, and in other cases they in using information derived from the methodology and not the data. The result is a loss of empirical content (McAllister, 1996).

Opponents and advocates agree that a posteriori methods, BPA and CCA (Zandee and Roos, 1987), permit no information (or components) other than present in those cladograms to be deleted or added: 
"Unlike Assumption 1 and 2 [applied in TAS, PSA, RTA and $\mathrm{CA}$ ], Assumption 0 [applied in BPA and CCA] does not allow for any taxon or area to be discarded at any time during the analysis." (Ebach, 1999, p. 388).

"Assumption 0 follows Hennig's auxiliary principle and does not allow for any removal of information" (Ebach, 2001, p. 386).

This fundamental difference between a priori and a posteriori methods gives the latter superior empirical content (sensu McAllister, 1996).

BPA is also superior to TreeMap on the basis of McAllister's criterion of novel predictions. BPA does not assume co-speciation a priori and thus each putative case of co-speciation discovered is a novel prediction; no co-speciation event supported by TreeMap can be a novel prediction, because the method assumes maximum co-speciation a priori. In addition, some advocates of the maximum co-speciation program have claimed to have developed novel insights into phylogenetic aspects of co-evolution. Examination of seven of those claims in light of the published records shows that six of them were first formulated by advocates of BPA, and the 7th, while discovered by advocates of TreeMap, represents yet another case in which BPA excels over TreeMap:

1. Parasites as synapomorphies of host clades: originally proposed by Hennig (1966), reiterated by Brooks (1979, 1981, figs 17-22), and used by Wiley (1986, 1988a,b) as the basis for Assumption 0. Reinvented without attribution by Clayton et al. (2003) and Johnson et al. (2003). Parenthetically, when TreeMap eliminates members of this class of widespread parasites, it eliminates evidence of co-speciation events.

2. Persistent ancestral parasites/sympatric speciation of parasites: first discussed explicitly by Brooks (1981, figs 39-44). Reinvented without attribution as lineage duplications by Page (1994a), who claimed that BPA could not recognize such events.

3. Using biogeographic information to test co-evolutionary scenarios: asserted by Hennig (1966) and made an explicit part of BPA by Brooks (1981), who later (Brooks, 1985) applied BPA to historical biogeography, following a suggestion by Arnold Kluge. Page (1994a) claimed to have originated the idea that phylogenetic methods of co-evolution and historical biogeography were different aspects of a common analytical problem. Clayton et al. (2003) and Siddall and Perkins also claimed this as an innovation of the maximum co-speciation model.

4. Duplicating hosts: originally proposed by Brooks (1990) to remove inclusive ORing from BPA. Partially reinvented without attribution by Siddall and Perkins.

5. Non-basal parasites in basal hosts: first discussed by Mitter and Brooks (1983) as a possible explanation for a major pattern of host distributions for phytophagous insects. Discussed explicitly as one class of problems for original formulation of BPA by Brooks and McLennan (1991, 2002). Empirical examples showing this "back colonization" phenomenon in systems of vertebrates and their parasites were presented by Hoberg and Adams (1992, 2000) and Hoberg (1995) and its detection by BPA were discussed by Brooks and McLennan (1991, 1993, 2002) and Hoberg et al. (1997). Experimental and phylogenetic studies, discussed at length in Brooks and McLennan (2002), have demonstrated that this pattern requires only a breakdown in allopatry coupled with exposure to an older member of the host clade, having the same plesiomorphic resource as the original host, to produce young parasites in old hosts. Reinvented without attribution by Siddall and Perkins.

6. Peripheral isolates speciation and non-parasitized members of a host clade: discussed explicitly in Brooks (1979, 1981) (see Figs 13, 14). Reinvented without attribution by Paterson and Gray (1997) as "missing the boat".

7. Host switches between sister taxa. Siddall and Perkins claimed that this is "methodologically impossible for BPA to yield." and then presented an example showing BPA yielding precisely that solution.

BPA results are also more consistent with the published record (for a representative sampling of the literature, see Brooks and McLennan, 1993, 2002; Hoberg and Klassen, 2002; Dowling et al., 2003), even including the small number of examples in Tangled Trees (Page, 2003b), which show substantial departures from maximum co-speciation, and which would therefore be more parsimoniously described by BPA.

Finally, because BPA is superior to TreeMap in terms of all the rest of McAllister's criteria, we conclude that BPA also has superior explanatory power to TreeMap. This means that BPA is superior to TreeMap on all grounds delineated by McAllister (1996), with the possible exception of aesthetic considerations at the ontological level. That is, some researchers simply find the idea that most co-evolution has been co-speciation to be more pleasing than the idea that co-evolution has been historically contingent with respect to host-parasite relationships.

Siddall and Perkins' dual assertion that BPA is deficient because it is not based on a model and because it is a likelihoodist exercise, is an example either of general confusion or of Orwellian rhetorical style. In the context of their attack on Dowling's study, they portray likelihood methods in a negative light, although this has not prevented either of them from using these methods in their own work (Siddall et al., 2001, 2004; Perkins and Schall, 2002), including support for Jungles, which is a likelihood model (Charleston, 1998). 


\section{Conclusions: ending a decade of deception}

The fundamental technical problems with primary BPA were identified within two years of its proposal (Cressey et al., 1983), and were solved by a combination of new computer technology (allowing missing data codes) (Wiley, 1986, 1988a,b), formulation of Assumption 0 (Wiley, 1986, 1988a,b; Zandee and Roos, 1987), and elimination of inclusive ORing (Brooks, 1990). The resulting version of BPA has been discussed at length in numerous texts, book chapters, and technical articles since 1990. Component 1.5 was released in 1990 , abandoned in 1993 for Component 2.0, which was abandoned in 1994 for RTA, which was abandoned in 1994 for TreeMap 1.0, which was abandoned in 2003 for TreeMap 2.02, and to which Siddall and Perkins' "fix" has now been added. All of these modifications have made Component Analysis more like, but not quite as good as, BPA.

It is tempting to ascribe this misrepresentation to ignorance and poor scholarship, except that each of Siddall and Perkins' mistakes had the effect of making it appear that BPA was deficient and TreeMap superior. Moreover, their article was not an isolated incident. The development and promotion of TreeMap has long been associated with attacks on BPA based on misrepresentations of its properties (chronology in Dowling et al., 2003). This has had wide-ranging effects. Studies purporting to compare BPA with other methods (Page, 1993, 1994a,b, 1995, 2003b,c; Morrone and Carpenter, 1994; Van Soest and Hajdu, 1997; Ebach, 2001; Ebach and Humphries, 2002) have used only the original formulation of BPA. A review of methods of historical biogeographic analysis (Crisci, 2001) did not mention BPA. An edited volume about comparative phylogenetic studies of co-evolution (Page, 2003b) contained repeated misrepresentations of BPA (Charleston and Perkins, 2003; Hafner et al., 2003; Johnson and Clayton, 2003; Paterson et al., 2003; Ronquist, 2003) and most recently, a monographic study of avian mites dismissed BPA in a single sentence, stating that there had been no developments in BPA since Brooks (1988) and that BPA had been replaced by TreeMap (Dabert, 2003).

Page (2003c) recently suggested that Brooks and McLennan (2002) had "missed the boat" by supporting a research program relying more on empirical data than models for explanations. If we empiricists have missed the boat, it was named Titanic, and the modifications of TreeMap designed to make it mimic BPA amount only to so much rearranging of the deck chairs. Advocates of maximum co-speciation have been insensitive to the growth of empirical data, and this has led to their models becoming progressively less relevant to coevolutionary studies. An extensive body of experimental and phylogenetic studies during the past decade all suggesting that there is no simple $1: 1$ mapping between the host resources underlying host specificity and host phylogeny; rather, the resources are generally phylogenetically conservative, making it possible for distantly related hosts to be inhabited by the same or related parasites due to their possession of a resource that is symplesiomorphic. This includes the occurrence of young parasites in old hosts, discussed first by Mitter and Brooks (1983), which excited Siddall and Perkins so much. Once again, BPA has priority. Brooks (1979) provided exemplars suggesting that narrowness of host specificity would not necessarily be a good criterion for expecting to find co-speciation. Subsequent experimental studies of the nature of host specificity have corroborated this assessment at macroevolutionary (Brooks and McLennan, 2002, Chap. 8) and microevolutionary (Thompson, 1994) levels.

The more tangled the trees, the more TreeMap must be modified, converging increasingly on BPA, because the core hypothesis of maximum co-speciation is simply wrong. Or, as Van Veller and Brooks (2001) put it, simplicity is not always the most parsimonious explanation of the real world. The original formulation of BPA was a valiant failure, for reasons first noted in 1983 and fixed by 1990 . Siddall and Perkins's own exemplars, like the exemplar presented by Ebach and Humphries (2002), corroborate the findings of Van Veller et al. (1999, 2000, 2001) and Dowling (2002) that all the modifications of a priori methods up to, and including TreeMap 2.02 have produced something almost, but not quite, as good as the original formulation of BPA. It may be understandably human to find it difficult to accept falsification of a model in which so much time, energy, and emotion have been invested, but it is ultimately irrelevant to the real issue. The advocates of the maximum co-speciation and maximum vicariance research programs have spent more than a decade striving, knowingly or unknowingly, to produce a method that was already in existence, and to claim credit for it. The simple assumptions of maximum vicariance and maximum co-speciation are beginning to sound not simple, but simple-minded, and it is time to move beyond them, using the full measure of modern phylogenetic inference to study the complexity of biological diversification. The failure of the maximum co-speciation program is rendered less than valiant by the fact that the modifications, such as they are, have been achieved more than a decade after the original formulation of BPA was replaced by the current version of BPA, the true success story.

\section{Acknowledgments}

We are indebted to Arnold Kluge for his insistence on honesty and accuracy in basic scholarship. We also thank Deborah McLennan for comments on various 
iterations of the manuscript. This study was supported in part by a grant from the Natural Sciences and Engineering Research Council (NSERC) of Canada to D.R.B.

\section{References}

Brooks, D.R., 1979. Testing the context and extent of host-parasite coevolution. Syst. Zool. 28, 299-307.

Brooks, D.R., 1981. Hennig's Parasitological method: a proposed solution. Syst. Zool. 30, 229-249.

Brooks, D.R., 1985. Historical ecology: a new approach to studying the evolution of ecological associates. Ann. Missouri Bot. Gard. $72,660-680$.

Brooks, D.R., 1988. Macroevolutionary comparisons of host and parasite phylogenies. Annu. Rev. Ecol. Syst. 19, 235-259.

Brooks, D.R., 1990. Parsimony analysis in historical biogeography and coevolution: methodological and theoretical update. Syst. Zool. 39, 14-30.

Brooks, D.R., 2003. The new orthogenesis. Cladistics, 19, 443-448.

Brooks, D.R., McLennan, D.A., 1991. Phylogeny, Ecology and Behavior: A Research Program in Comparative Biology. University of Chicago Press, Chicago, IL.

Brooks, D.R., McLennan, D.A., 1993. Parascript: Parasites and the Language of Evolution. Smithsonian Institution Press, Washington, DC.

Brooks, D.R., McLennan, D.A., 2002. The Nature of Diversity: An Evolutionary Voyage of Discovery. University of Chicago Press, Chicago, IL.

Brooks, D.R., McLennan, D.A., 2003. Extending phylogenetic studies of coevolution: secondary Brooks parsimony analysis, parasites, and the Great Apes. Cladistics, 19, 104-119.

Brooks, D.R., Van Veller, M.G.P., McLennnan, D.A., 2001. How to do BPA, really. J. Biogeogr. 28, 345-358.

Charleston, M.A., 1998. Jungles: a new solution to the host/parasite phylogeny reconciliation problem. Math. Biocsci. 149, 191-223.

Charleston, M.A., Perkins, S.L., 2003. Lizards, malaria, and jungles in the Caribbean. In: Page, R.D.M. (Ed.), Tangled Trees. University of Chicago Press, Chicago, IL, pp. 65-92.

Clayton, D.H., Al-Tamimi, S., Johnson, K.P., 2003. The ecological basis of coevolutionary history. In: Page, R.D.M. (Ed.), Tangled Trees. University of Chicago Press, Chicago, IL, pp. 65-92.

Cressey, R.F., Collette, B., Russo, J., 1983. Copepods and scombrid fishes: a study in host parasite relationships. Fish. Bull. 81, 227265.

Crisci., J., 2001. The voice of historical biogeography. J. Biogeogr. 28, $157-168$.

Dabert, J., 2003. The feather family Syringobiidae Trouessart. 1896 (Acari, Astigmata, Pterlichoidea). Acta Parasitologica Suppl. 48, S1-S233.

Dowling, A.P.G., 2002. Testing the accuracy of TreeMap and Brooks parsimony analyses of coevolutionary patterns using artificial associations. Cladistics, 18, 416-435.

Dowling, A.P.G., van Veller, M.G.P., Hoberg, E.P., Brooks, D.R., 2003. A priori and a posteriori methods in comparative evolutionary studies of host-parasite associations. Cladistics, 19, 240-253.

Ebach, M.C., 1999. Paralogy and the centre of origin concept. Cladistics, 13, 387-391.

Ebach, M.C., 2001. Extrapolating cladistic biogeography: a brief comment on van Veller et al. (1999, 2000, 2001). Cladistics, 17, 383-388.

Ebach, M.C., Humphries, C.J., 2002. Cladistic biogeography and the art of discovery. J. Biogeogr. 29, 427-444.

Ebach, M.C., Humphries, C.J., Williams, D.M., 2003. Phylogenetic biogeography deconstructed. J. Biogeogr. 30, 1285-1296.
Green, M., Van Veller, M.G.P., Brooks, D.R., 2002. Assessing modes of speciation: range asymmetry and biogeographical congruence. Cladistics, 18, 112-124.

Hafner, M.S., Demastes, J.W., Spradling, T.A., Reed, D.L., 2003. Cophylogeny between pocket gophers and chewing lice. In: Page, R.D.M. (Ed.), Tangled Trees. University of Chicago Press, Chicago, IL, pp. 221-239.

Hennig, W., 1966. Phylogenetic Systematics. University of Illinois Press, Urbana, IL.

Hoberg, E.P., 1986. Evolution and historical biogeography of a parasite-host assemblage: Alcataenia spp. (Cyclophyllidea: Dilepididae) in Alcidae (Charadriiformes). Can. J. Zool. 64, 25762589.

Hoberg, E.P., 1995. Historical biogeography and modes of speciation across high latitude seas of the Holarctic: concepts for host-parasite coevolution among the Phocini (Phocidae) and Tetrabothriidae (Eucestoda). Can. J. Zool. 73, 45-57.

Hoberg, E.P., Adams, A.M., 1992. Phylogeny, historical biogeography and ecology of Anophryocephalus spp. (Eucestoda: Tetrabothriidae) among pinnipeds of the Holarctic during the late Tertiary and Pleistocene. Can. J. Zool. 70, 703-719.

Hoberg, E.P., Adams, A., 2000. Phylogeny, history and biodiversity: understanding faunal structure and biogeography in the marine realm. Bull. Scand. Soc. Parasitol. 10, 19-37.

Hoberg, E.P., Brooks, D.R., Siegel-Causey, D., 1997. Host-parasite phylogenies and cospeciation. In: Clayton, D., Moore, J. (Eds), Host-Parasite Evolution: General Principles and Avian Models. Oxford University Press, Oxford, UK, pp. 212-235.

Hoberg, E.P., Klassen, G.J., 2002. Revealing the faunal tapestry: co-evolution and historical biogeography of hosts and parasites in marine systems. Parasitology, 124, S3-S22.

Johnson, K.P., Adams, R.J., Page, R.D.M., Clayton, D.H., 2003. When do parasites fail to speciate in response to host speciation? Syst Biol. 52, 37-47.

Johnson, K.P., Clayton, D.H., 2003. Coevolutionary history of ecological replicates: comparing phylogenies of wing and body lice to columbiform hosts. In: Page, R.D.M. (Ed.), Tangled Trees. University of Chicago Press, Chicago, IL, pp. 262-286.

McAllister, J.W., 1996. Beauty and Revolution in Science. Cornell University Press, Ithaca, NY.

Mindell, D.P., Schultz, J.W., Ewald, P.W., 1995. The AIDS pandemic is new, but is HIV new? Syst. Biol. 44, 77-92.

Mitter. C., Brooks, D.R., 1983. Phylogenetic aspects of coevolution. In: Futuyma, D.J., Slatkin, M. (Eds.), Coevolution. Sinauer Associates, Sunderland, MA.

Morrone, J.J., Carpenter, J.M., 1994. In search of a method for cladistic biogeography: an empirical comparison of Component Analysis, Brooks Parsimony Analysis, and Three-area Statements. Cladistics, 10, 99-153.

Nelson, G., Ladiges, P.Y., 1991. Three-area statements: standard assumptions for biogeographic analysis. Syst. Zool. 40, 470-485.

Nelson, G., Ladiges, P.Y., 1996. Paralogy in cladistic biogeography and analysis of paralogy-free subtrees. Am. Mus. Novitates, 3167, $1-58$.

Nelson, G., Platnick, N.I., 1981. Systematics and Biogeography; Cladistics and Vicariance. Columbia University Press, New York/Guilford, UK.

Page, R.D.M., 1993. Component 2.0: Tree Comparison Software for Microsoft Windows. Program and User's Manual. Natural History Museum, London.

Page, R.D.M., 1994a. Maps between trees and cladistic analysis of historical associations among genes, organisms, and areas. Syst. Biol. 43, 58-77.

Page, R.D.M., 1994b. Parallel phylogenies: reconstructing the history of host-parasite assemblages. Cladistics, 10, 155-173.

Page, R.D.M., 1995. Treemap 1.0. Program and User's Manual. Division of Environmental and Evolutionary Biology, Institute of 
Biomedical and Life Sciences. University of Glasgow, Glasgow, UK.

Page, R.D.M., 2003a. Missing the boat. J. Evol. Biol. 16, 1064.

Page, R.D.M., 2003b. Treemap 2.02. Program and User's Manual. Division of Environmental and Evolutionary Biology, Institute of Biomedical and Life Sciences, University of Glasgow, Glasgow, UK.

Page, R.D.M., ed., 2003c. Tangled Trees. University of Chicago Press, Chicago, Illinois.

Paterson, A.M., Gray, R.D., 1997. Host-parasite co-speciation, host switching, and missing the boat. In: Clayton, D.H., Moore, J. (Eds.), Host-Parasite Evolution: General Principles and Avian Models. Oxford University Press, Oxford, UK, pp. 236-250.

Paterson, A.M., Palma, R.L., Gray, R.D., 2003. Drowning on arrival, missing the boat, and X-events: How likely are sorting events? In: Page, R.D.M. (Ed.), Tangled Trees. University of Chicago Press, Chicago, IL, pp. 287-309.

Perkins, S.L., Schall, J.J., 2002. A molecular phylogeny of malarial parasites recovered from cytochrome $b$ gene sequences. J. Parasitol. 88, 972-978.

Ronquist, F., 2003. Parsimony analysis of coevolving species associations. In: Page, R.D.M. (Ed.), Tangled Trees. University of Chicago Press, Chicago, IL, pp. 22-64.

Siddall, M.E., Apakupakul, K., Burreson, E.M., Coates, K.A., Erseus, C., Gelder, S.R., Kallersjo, M., Trapido-Rosenthal, H., 2001. Validating Livanow: Molecular data agree that leeches, branchiobdellidans, and Acanthobdella peledina form a monophyletic group of oligochaetes. Mol. Phylo. Evol. 21, 346-351.

Siddall, M.E., Perkins, S.L., 2003. Brooks Parsimony Analysis: a valiant failure. Cladistics, 19, 554-564.

Siddall, M.E., Perkins, S.L., Desser, S.S., 2004. Leech mycetome endosymbionts are a new lineage of alphaproteobacteria related to the Rhizobiaceae. Mol. Phylo. Evol. 30, 178-186.
Thompson, J., 1994. The Coevolutionary Process. University of Chicago Press, Chicago, IL.

Van Soest, R.W.M., Hajdu, E., 1997. Marine area relationships from twenty sponge phylogenies. A comparison of methods and coding strategies. Cladistics, 13, 1-20.

Van Veller, M.G.P., Brooks, D.R., 2001. When simplicity is not parsimonious: A priori and a posteriori methods in historical biogeography. J. Biogeogr. 28, 1-11.

Van Veller, M.G.P., Brooks, D.R., Zandee, M., 2003. Cladistic and phylogenetic biogeography: the art and the science of discovery. J. Biogeogr. 30, 319-329.

Van Veller, M.G.P., Kornet, D.J., Zandee, M., 2000. Methods in vicariance biogeography: assessment of the implementations of assumptions zero, 1 and 2. Cladistics, 16, 319-345.

Van Veller, M.G.P., Kornet, D.J., Zandee, M., 2002. A posteriori and a priori methodologies for testing hypotheses of causal processes in vicariance biogeography. Cladistics, 18, 207-217.

Van Veller, M.G.P., Zandee, M., Kornet, D.J., 1999. Two requirements for obtaining valid common patterns under different assumptions in vicariance biogeography. Cladisitcs, 15, 393-406.

Van Veller, M.G.P., Zandee, M., Kornet, D.J., 2001. Measures for obtaining inclusive solution sets under assumptions zero, 1 and 2 with different methods for vicariance biogeography. Cladistics, 17, 248-259.

Wiley, E.O., 1986. Methods in vicariance biogeography. In: Hovenkamp, P. (Ed.), Systematics and Evolution. University of Utrecht Press, Utrecht, Netherlands, pp. 283-306.

Wiley, E.O., 1988a. Parsimony analysis and vicariance biogeography. Syst. Zool. 37, 271-290.

Wiley, E.O., 1988b. Vicariance biogeography. Annu. Rev. Ecol. Syst, $19,513-542$.

Zandee, M., Roos, M.C., 1987. Component-compatibility in historical biogeography. Cladistics, 3, 305-332. 